\title{
Predictores de la Retención en una Comunidad Terapéutica para Drogodependientes
}

\author{
Secades Villa, R.; Magdalena Benavente, Y.
}

Departamento de Psicología. Universidad de Oviedo.

\section{RESUMEN}

El abandono de los tratamientos es uno de los principales problemas en el ámbito de la salud mental y se trata de un fenómeno particularmente importante en los programas por abuso de drogas. El objetivo de este estudio es evaluar las tasas de retención de una comunidad terapéutica para drogodependientes y conocer la influencia de las variables pre y durante el tratamiento sobre la permanencia de los pacientes en el programa. La tasa de abandonos fue del 32,7\% y la duración media de la permanencia de 7 meses. El estudio muestra que las variables relacionadas con los sujetos no predicen el abandono mientras que las variables relacionadas con el tratamiento estaban asociadas con una mayor retención. Se concluye que los eventos durante el tratamiento son cruciales para la retención de los pacientes en los tratamientos de drogodependencias.

Palabras clave: Retención, abandono, variables de los pacientes, variables del programa, comunidad terapéutica.

\section{INTRODUCCIÓN}

E I abandono del tratamiento es uno de los principales problemas en el ámbito de la salud mental y se trata de un fenómeno de especial gravedad en los tratamientos por abuso de drogas. En este tipo de programas las tasas de abandono son muy elevadas y, como ya apuntaron Baekeland y Lundwall (1975), son especialmente altas en los programas libres de drogas. Conclusiones similares se han venido observando en otros estudios clásicos posteriores como el de Simpson (1984) o el de Hubbard et al. (1984) y en trabajos más recientes como los de De leon (1991) o D'Ippoliti et al. (1998).

En España, varios autores se han preocupado también por estudiar el fenómeno del abandono, encontrándose, en general, resultados muy similares a los

\section{SUMMARY}

The problem of early attrition from treatment is pervasive throughout community mental health and is particularly high for substance abusing clients. The purpose of this study was to evaluate program completion data and to determine the effects of pre-and in-treatment variables on patient retention in a therapeutic community for substance abusers. The rate of dropouts was $32,7 \%$ and the estimated median treatment duration was 7 months. Research shows that client variables do not predict dropouts and the treatment variables were associated with longer retention in treatment. We concluded that events during treatment are crucial for patient retention in treatment for substance abusers.

Key words: retention, dropout, client variables, treatment variables, therapeutic community.

anteriormente mencionados (por ejemplo, SánchezCarbonell, Camí y Brigos, 1988; Ochoa et al.,1992; Machado y Girón, 1993; Gutierrez et al., 1995; Torrens, Castillo y Pérez-Sala, 1996; Secades, Fernández Rodríguez y Fernández Hermida, 1998).

En definitiva, todos los estudios de seguimiento sobre programas dirigidos a drogodependientes refieren tasas de abandono muy altas. Estas tasas son menores en los tratamientos farmacológicos y más elevadas en los programas libres de drogas.

Las consecuencias de este fenómeno en el ámbito de las drogodependencias son muy graves, ya que la mayoría de los abandonos suponen un mal pronósti$\mathrm{co}$, sobre todo si éstos se producen en los primeros momentos del tratamiento.

Por otro lado, el tipo de salida del programa también se relaciona con el efecto que ejerce éste sobre 
la conducta de los sujetos a largo plazo. Así, revisiones como las de Tucker, Vuchinich y Harris (1985) y Stark (1992) concluyen de una forma muy clara que los sujetos que terminan los tratamientos de abuso de alcohol y otras drogas tienen muchos mejores resultados que los que abandonaban. Es decir, mantenerse en el tratamiento y terminarlo tiene un potente valor predictivo sobre los resultados.

Factores relacionados con el abandono del tratamiento

La literatura científica sobre los factores relacionados con la retención suele recoger dos tipos o grupos de variables, con especial énfasis en las primeras. Por una parte, las variables de los pacientes, en donde se suelen incluir las características de personalidad del sujeto, aspectos picopatológicos, variables sociodemográficas, estado de salud, incidencias legales, historia de consumo y motivación para el tratamiento. Por otra, las variables relacionadas con el tratamiento, como el tipo y las características del programa, los procedimientos empleados o la evolución del paciente en el mismo.

Las investigaciones que tratan de buscar características de los pacientes relacionadas con la retención han obtenido resultados dispares, por lo que se puede afirmar que este tipo de factores no son buenos pre- dictores de la retención (Condelli, 1994). Quizas habría que matizar esta conclusión señalando dos excepciones. En primer lugar, la relación entre abandono y presencia de trastornos psicopatológicos ofrece conclusiones diferentes. En general, una mayor severidad en este tipo de trastornos se relaciona con una menor permanencia en los programas de tratamiento $y$, en general, con un peor pronóstico.

Por otra parte, existe cierta consistencia en cuanto a que las variables disposicionales (en general, motivación hacia el tratamiento) han resultado ser factores importantes para la retención en diferentes tipos de programas (Erickson, Stevens, Mcknight y Figueredo, 1995; De León, Melnick y Kressel, 1997; Melnick, De León, Hawke, Jainchill y Kressel, 1997; Joe, Simpson y Broome, 1998; González-Sáiz et al., 1998).

Por lo que se refiere a la relación entre los factores del tratamiento y la retención, los resultados apuntan a que este tipo de variables parecen ser predictores más potentes y consistentes del abandono (Condelli, 1994; Magura, Nwakeze y Demsky, 1998). En concreto, las siguientes condiciones parecen favorecer una mayor retención: estabilidad de los terapeutas, participación de la familia, programa residencial frente al ambulatorio, tratamiento farmacológico (sobre todo con metadona) frente a los programas libres de drogas y dosis altas frente a dosis bajas del fármaco (Tabla 1).

Tabla 1. Factores del tratamiento relacionados con el abandono

\begin{tabular}{ll}
\hline Variable & Estudios \\
\hline Estabilidad de los terapeutas & Craig, Rogalski y Veltri (1982), McCaul y Svikis (1991), \\
& Caballero (1993) \\
\hline Apoyo familiar & Panyard, Wolf y Dreachslin (1979), Simpson (1979), \\
& Stanton et al. (1984), De Leon (1991), Sisson y Azrin (1986), \\
& Higgins et al.(1994), Ochoa, Cebollada e Ibáñez (1994) \\
& Torrens, Castillo y Pérez-Sola (1996), Conner et al. (1998), \\
& Secades, Fernández Rodríguez y Fernández Hermida (1998) \\
\hline Contexto terapéutico (residencial/ambulatorio) & Alterman et al. (1994), Bell et al. (1994), Roberts y \\
& Nishimoto (1996), D'Ippoliti et al. (1998) \\
\hline
\end{tabular}

Tipo de tratamiento:

Carroll et al. (1998), Maremmani et al. (1994)

farmacológico/libre de drogas

Dosis del fármaco

Torrens Castillo y Pérez-Sola (1996), Del Río, Mino y

Perneger (1997), Magura, Nwakeze y Demsky (1998)

No obstante, a pesar de que los factores relacionados con el tratamiento parecen ser mejores predictores de la retención que las características de los pacientes, son pocos los estudios en donde se hayan incluído variables del primer grupo (Condelli, 1994).

Con este trabajo se pretende, en primer lugar, conocer las tasas de retención/abandono de una Comunidad Terapéutica para drogodependientes. En segundo lugar, conocer si determinadas variables (individuales y del tratamiento) determinan la permanencia o el abandono de los pacientes en este programa.

El estudio de las tasas de retención y de los factores que están afectando al abandono de los sujetos nos orientaría hacia la implantación de estrategias terapéuticas que incrementasen la probabilidad de 
permanencia de los pacientes y, por tanto, el descenso en el consumo de drogas.

\section{MÉTODO}

\section{Sujetos}

Se ha utilizado una muestra de 52 sujetos drogodependientes que ingresaron en una Comunidad Terapéutica profesional del Principado de Asturias.

Todos eran varones y politoxicómanos, aunque su droga de consumo principal era la heroína, motivo por el que asistieron al programa.

Algunas características de los sujetos en el momento del ingreso en el programa de tratamiento aparecen en la Tabla 2.

\section{Variables}

Los datos han sido obtenidos a partir de las historias clínicas (autoinformes) y de los diferentes instrumentos de observación y recogida de datos del centro.

Variables predictoras de los sujetos:

- Variables sociodemográficas:

(1) Edad, (2) Estado Civil, (3) Situación Laboral, (4) Nivel de estudios, (5) Personas con las que convive en el momento del ingreso.

- Variables de la historia de consumo:

(6) Número de drogas en su historia de consumo, (7) Edad de inicio en el alcohol, (8) Edad de inicio en el hachís, (9) Edad de inicio en la heroína, (10) Años de consumo de heroína, (11) Dosis diaria de heroína en el último año, (12) Vía de administración de la heroína antes del ingreso, (13) Número de intentos de dejar la heroína (al menos una semana de abstinencia), (14) Tiempo máximo de abstinencia (en semanas).

- Variables médicas y judiciales:

(15) Enfermedades derivadas del consumo, (16) Incidencias legales posteriores al consumo, (17) Ingresos en prisión, (18) Juicios pendientes.

- Antecedentes familiares de consumo de drogas:
(19) Antecedentes familiares de consumo de heroína, (20) Antecedentes familiares de consumo de alcohol.

- Variables "disposicionales":

(21) Motivo principal para acudir al tratamiento, (22) Autoeficacia ante situaciones de riesgo, valorada con el Inventory of Drug-Taking Situations (IDTS) (Annis, 1985), (23) Expectativas hacia el tratamiento.

Variables predictoras del tratamiento:

(1) Número de sanciones impuestas a lo largo del programa, (2) Número de salidas realizadas al domicilio particular, (3) Tiempo total fuera del centro durante el programa (en días), (4) Consumos de alcohol durante el tratamiento, (5) Consumos de hachís durante el tratamiento, (6) Consumos de heroína durante el tratamiento, (7) Procedencia de los pacientes (vía privada o derivado de otro programa), (8) Tasas de objetivos cumplidos en las salidas, (9) Cursos de formación realizados durante el tratamiento, (10) Afrontamiento de situaciones de riesgo durante las salidas (valoración del terapeuta), (11) Manejo del dinero durante las salidas (valoración del terapeuta), (12) Aficiones durante el tratamiento, (13) Apoyo familiar.

Variable criterio:

La variable criterio es la permanecia o el abandono de los pacientes en el programa. Esta variable se ha operativizado en otras dos:

(1) Tipo de salida del programa: alta terapéutica/ abandono.

(2) Número de meses de permanencia en el programa.

\section{RESULTADOS}

Los procedimientos estadísticos utilizados han sido el análisis discriminante (método stepwise) que nos permite averiguar la capacidad predictiva de las variables sobre el tipo de salida, y la regresión múltiple, con el fin de conocer la capacidad predictiva de las variables del estudio sobre el número de meses de permanencia en el programa. Se han realizado ambos tipos de análisis incluyendo por separado como variables predictoras las características de los sujetos y las relacionadas con el tratamiento.

Tabla 2. Características sociodemográficas de la muestra ( $N=52$ )

\begin{tabular}{llll}
\hline Edad & Estado civil & Nivel de estudios & Situación laboral \\
\hline Media: 30 & Solteros: $75 \%$ & Sin estudios: $16,7 \%$ & En paro:67\% \\
Rango: $21-40$ & Casados: $11,5 \%$ & EGB: $51,1 \%$ & Baja o ILT: 29\% \\
& Separados: $13,5 \%$ & Estudios medios: $29 \%$ & Pensionado: $4 \%$
\end{tabular}


El paquete estadístico utilizado ha sido el SPSS+, versión 8.0.

La tasa global de abandonos del programa es de $32,7 \%$ (es decir, 17 sujetos de los 52 de que consta la muestra). La media de estancia ha sido de 28 semanas ( 7 meses), con un rango entre 8 y 56 semanas (entre 2 y 14 meses). La mayoría de los abandonos $(64,6 \%)$ ocurren durante los cuatro primeros meses de tratamiento y la cifra va decreciendo a medida que transcurre el tiempo de permanencia (entre los cinco y los nueve meses abandona el $35,3 \%$ de los sujetos) (Figura 1).

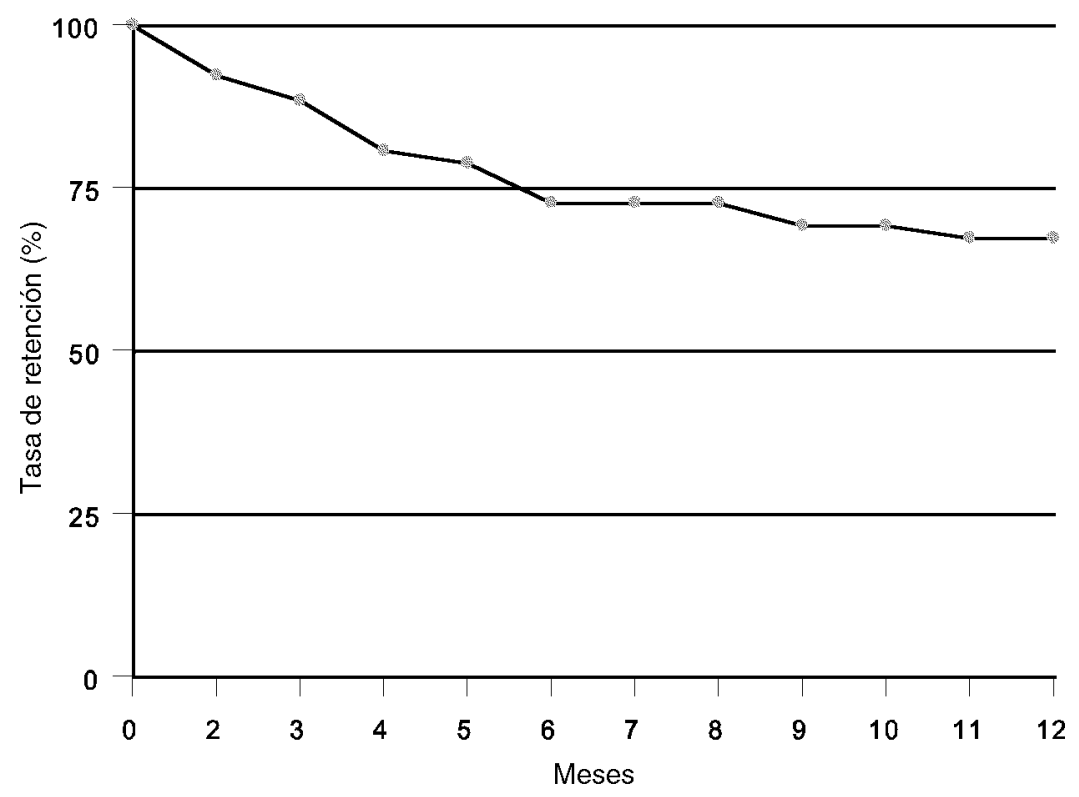

Figura 1. Curva de retención del programa

Los resultados de los análisis discriminantes realizados con todas las variables individuales conjuntamente y los realizados con los grupos de variables por separado (sociodemográficas, historia de consumo, médicas, judiciales, antecedentes familiares y disposicionales) muestran que estas variables no tienen ninguna capacidad predictiva para determinar el tipo de salida del programa (alta o abandono) de un paciente.

En cambio, el análisis discriminante realizado con las variables relacionadas con el tratamiento ha mostrado que éstas poseen una capacidad predictiva considerable $(82,7 \%)$ (Tabla 3).

Los resultados de este análisis muestran que las variables: aficiones durante el tratamiento, consumos de heroína durante el tratamiento y familiares que prestan apoyo durante el programa son

Tabla 3. Capacidad predictiva de las variables de tratamiento (Análisis Discriminante)

\begin{tabular}{lccc}
\hline Grupo & Casos & $\mathbf{1}$ & $\mathbf{2}$ \\
\hline Alta terapéutica (1) & 35 & $34(97,1 \%)$ & $1(2,9 \%)$ \\
Abandono (2) & 17 & $8(47,1 \%)$ & $9(52,9 \%)$ \\
\hline
\end{tabular}

Porcentaje de casos correctamente clasificados: $82,7 \%$ variables que discriminan adecuadamente el tipo de salida en un $82,7 \%$ de los casos. Es importante señalar que el consumo de heroína durante el transcurso del programa no conllevaba una expulsión del mismo.

En lo que se refiere a los resultados de los análisis de regresión múltiple realizados con la variable criterio "número de meses de permanencia en el programa" y utilizando como variables predictoras, por una parte, el conjunto de las variables individuales $y$, por otra, los diferentes grupos de variables, muestran que estas variables no tienen capacidad predictiva suficiente para determinar el tiempo de permanencia en el programa de un paciente. Únicamente, el análisis realizado con las variables de la historia de consumo dio como resultado que la edad de inicio en el consumo de heroína poseía una cierta capacidad predictiva, pero esta, no obstante, era muy poco significativa $\left(\mathrm{R}^{2}=, 139\right)$.

Sin embargo, los resultados del análisis de regresión realizado con las variables de tratamiento vuelven a mostrar que este grupo de variables, en concreto, el tiempo total de salidas del centro y los cursos de formación realizados durante la estancia en éste poseen una capacidad predictiva considerable sobre el tiempo de permanencia, en concreto el 66,5\% (Tabla 4). 
Tabla 4. Resultados de la Regresión Múltiple (variables del tratamiento)

\begin{tabular}{lcrrr}
\hline Variables & $\mathbf{R}$ & $\mathbf{R}^{2}$ & $\begin{array}{c}\mathbf{R}^{2} \\
\text { ajustada }\end{array}$ & $\mathbf{F}$ \\
\hline Tiempo & & & & \\
total de salidas & $787^{\mathrm{a}}$ &, 619 &, 608 &, 000 \\
Cursos realizados &, $816^{\mathrm{b}}$ &, 665 &, 645 &, 038 \\
\hline
\end{tabular}

\section{DISCUSIÓNY CONCLUSIONES}

El objetivo principal de este estudio era conocer las variables que predecían el abandono o la permanencia en una Comunidad Terapéutica para drogodependientes. El conocimiento de la relación entre las variables objeto de estudio y la retención nos permitiría mejorar las tasas de permanencia del programa y, por tanto, reducir el número de recaídas de los pacientes.

La tasa de abandono global encontrada en el programa se sitúa en el 32,7\%. Esta cifra es significaticamente inferior a la media encontrada en otros estudios realizados en programas similares (SánchezCarbonell et al., 1988; De Leon, 1991; D'Ippoliti et al., 1998). Por tanto, si uno de los criterios para medir la eficacia del tratamiento es la capacidad de retención, podríamos decir que la Comunidad Terapéutica en donde se ha realizado este estudio presenta, en este aspecto, unos resultados muy positivos.

La mayoría de los abandonos ocurren en las primeras fases del tratamiento. Estos datos son coincidentes con los resultados de otros trabajos (Baekelend y Lundwall, 1975; De Leon, 1991; Kooyman, 1993/1996; Secades, Fernández Rodríguez y Fernández Hermida, 1998). Es conocido que estos abandonos son los más graves (los que tienen peor pronóstico) y que la probabilidad de una permanencia continuada en el programa aumenta considerablemente después de 90 días. Esto es, la probabilidad de abandono decrece con el paso del tiempo. Así, la representación gráfica de las curvas de retención da lugar a un patrón característico en forma de hipérbola, que es el mismo para todos los programas (sobre todo los libres de drogas), aunque difieren en las tasas absolutas de retención.

Por tanto, se hace necesario recalcar la importancia que tiene mejorar el ajuste de los pacientes en las fases tempranas de máxima vulnerabilidad, por medio de estrategias específicas que incrementen las tasas de retención en estas primeras semanas.

En cuando a los resultados de los análisis multivariados, éstos han mostrado que los eventos que ocurren durante el tratamiento son cruciales para determinar la retención. Estos datos son coincidentes con las conclusiones de otros estudios recientes (por ejemplo, Condelli, 1994; Magura et al., 1998; Morral, Belding e lguchi, 1999).

Así, los resultados de los análisis discriminantes (tanto del análisis del conjunto de variables como el realizado por grupos) indican que las variables con mayor capacidad pronóstica son algunas de las relacionadas con el tratamiento. En concreto: aficiones durante el tratamiento, consumos de heroína durante el tratamiento y familiares que prestan apoyo durante el programa.

Los sujetos que permanecen más tiempo en el programa son aquellos que cuentan con más aficiones, es decir, que ocupan mejor su tiempo libre y organizan de manera más eficaz los momentos en los que no hay actividades programadas durante el tratamiento.

El consumo de heroína durante el tratamiento ha sido otra variable determinante de la retención. Este resultado coincide con los de otros estudios en donde se han tenido en cuenta este tipo de factores. En concreto, Magura et al. (1998) encontraron que el uso de heroína y cocaína de los pacientes en un programa de metadona incrementaba la probabilidad del abandono de éstos; mientras que los resultados de Morral, Belding e Iguchi (1999) señalaron que sólo dos variables, la asistencia a las terapias y la abstinencia de opiáceos en las primeras fases del programa pronosticaban más del $80 \%$ de los abandonos.

Por otra parte, la implicación activa de la familia en el proceso terapéutico funciona también como un factor protector frente al abandono prematuro. El efecto beneficioso de la colaboración familiar sobre la evolución de los pacientes en los programas de tratamiento ya cuenta con una amplia evidencia empírica (por ejemplo, Conner et al., 1998).

Esta tendencia (la mayor influencia de los procesos del tratamiento sobre la retención) se ve confirmada con el análisis de regresión, en donde las variables de mayor capacidad pronóstica son el tiempo total de salidas y los cursos de formación realizados durante el tratamiento. Llama la atención el hecho de que los sujetos que más días tienen de salida son los que mejor resultado obtienen en el tratamiento. Se puede creer que este resultado podría estar contradiciendo uno de los postulados fundamentales de los programas residenciales, en concreto, el del aislamiento del sujeto. No obstante, se debe pensar que los sujetos con más tiempo de salidas son aquellos que cumplen mejor la normativa del centro y más progresan en su rehabilitación, por lo que es lógico que obtengan mejores resultados. En definitiva, las salidas se verían como indicador de la evolución del paciente en cuanto a la generalización de los aprendizajes, el restablecimiento de las relaciones familiares y la búsqueda de 
nuevas relaciones interpersonales y alternativas al consumo de drogas.

La variable "cursos de formación realizados durante el tratamiento" se encuentra muy relacionada con la variable "aficiones durante el tratamiento" comentada con anterioridad. Estos resultados indican el efecto beneficioso de cubrir de manera positiva el tiempo libre y de preparar al sujeto para una futura reinserción social también en este aspecto.

En general, podríamos calificar estos datos como positivos o prometedores, en cuanto que son este tipo de variables las que estan más sujetas a una modificación por parte de los clínicos, en contraste con otros factores estáticos (como los sociodemográficos o los de historia de consumo), en los que no es posible realizar ningún cambio.

En definitiva, de los resultados extraidos de este estudio se pueden extraer varias consideraciones de orden práctico:

1. En primer lugar, la necesidad de implementar intervenciones específicas en el inicio de los programas de tratamiento (cuando las tasas de abandono son mayores) con el fin de incrementar la motivación de los pacientes y, por tanto, reducir las tasas de abandono prematuro.

2. Las variables relacionadas con el tratamiento son mejores predictores de la retención que las relacionadas con las características individuales.

3. En concreto, es importante que en el diseño de los programas con drogodependientes se tenga en cuenta la inclusión de intervenciones orientadas hacia el uso alternativo del ocio y tiempo libre, así como actividades de formación y orientación educativa y/o profesional y la inclusión de la posibilidad de salidas al ambiente natural de los pacientes con el fin de poner en práctica los recursos y las habilidades adquiridas durante el tratamiento.

4. Una vez más se debe resaltar la necesidad de implicar a la familia en la rehabilitación del paciente de forma activa.

5. Por último, se hace necesario la realización de investigaciones centradas en la relación entre los factores del tratamiento y la retención, frente a aquellas que sólo tienen en cuenta las características de los pacientes.

\section{AGRADECIMIENTOS}

Los autores desean agradecer sinceramente a los responsables de la Comunidad Terapéutica "El Valle" en Tuñón (Asturias) su indispensable colaboración para la realización de este trabajo.

\section{REFERENCIAS BIBLIOGRÁFICAS}

Alterman, A.I., O'Brien, C.P., McLellan, T., August, D.S., Snider,E.C., Droba, M., Cornish, J.W., Hall, C.P., Raphaelson, A.H. y Schrade, F.X. (1994). Effectiveness and costs of impatient versus day hospital cocaine rehabilitation. Journal of Nervous and Mental Disease, 152, 157-163.

Annis, H.M. (1985). Inventory of Drug-Taking Situations. Toronto: Addiction Research Foundation.

Baekeland, F. y Lundwall, L. (1975). Dropping out of treatment: A critical review. Psychological Bulletin, 5, 738783.

Bell, D.C., Williams, M.L., Nelson, R. y Spence, R.T. (1994). An experimental test of retention in residential and outpatient programs. American Journal of Drug and Alcohol Abuse, 20, 331-340.

Caballero, L. (1993). Determinantes y significados de la retención en programas para drogodependientes. En J. Sinesquillas (Dir.), Recaída y prevención de recaídas (pp.243-266). Sitges: Ediciones en Neurociencias.

Conner, K.R., Shea, R.R., McDermott, M.P., Grolling, R., Tocco, R.V. y Baciewicz, G. (1998). The role of multifamily therapy in promoting retention in treatment of alcohol and cocaine dependence. American Journal of Addiction, 7, 61-73.

Condelli, W.S. (1994). Domains of variables for understanding and improving retention in therapeutic communities. International Journal of the Addictions, 29, 593-607.

Conner, K.R., Shea, R.R., McDermott, M.P., Grolling, R., Tocco, R.V. y Baciewicz, G. (1998). The role of multifamily therapy in promoting retention in treatment of alcohol and cocaine dependence. American Journal of Adicctions, 7, 61-73.

Craig, R.J., Rogalski, C. y Veltri, D. (1982). Predicting treatment dropouts from a drug abuse rehabilitation program. International Journal of the Addictions, 4, 641-653.

De Leon, G. (1991). Retention in drug-free therapeutic communities. En R.W. Pickens, C.G. Leukefeld y C.R. Schuster (Eds.), Improving drug abuse treatment. NIDA Research Monograph Series, $n^{\circ}$ 106. Rockville, MD: US Department of Health and Human Services.

De Leon, G., Melnick, G. y Kressel, D. (1997). Motivation and readiness for therapeutic community treatment among cocaine and other drug abusers. American Journal of Drug and Alcohol Abuse, 23, 169-189.

Del Rio, Mino, A. y Perneger, T.V. (1997). Predictors of patient retention in a newly established methadone maintenance treatment programme. Addiction, 92, 1353-1360.

D'Ippoliti, D., Davoli, M., Perucci, C.A., Pasqualini, F. y Bargagli, A.M. (1998). Retention in treatment of heroin users in Italy: the role of treatment type and of methadone maintenance dosage. Drug and Alcohol Dependence, 52, 167-171. 
Erickson, J.R., Stevens, S., McKnight, P. y Figueredo, A.J. (1995). Willingness for treatment as a predictor of retention and outcomes. Journal of Addictive Diseases, 14, 135-150.

González Sáiz, F., De Leon, G., Macías, C.G., Lillo, P., Varela, L., Castro, E., Gómez, E., Ruiz, N., Montañés, M. y Salvador-Carulla, L. (1998). La motivación y las actitudes ante el tratamiento como predictores de abandono precoz en Comunidad terapéutica: datos preliminares de la adpatación de la escala CMRS. V Encuentro Nacional sobre drogodependencias y su Enfoque Comunitario. Cádiz.

Gutiérrez, M., Ballesteros, J., González-Oliveros, R. y Ruiz de Apodaka, J. (1995). Tasa de retención en dos programas de naltrexona para heroinodependientes en Vitoria, España. Revista de Psiquiatría de la Facultad de Medicina de Barcelona, 22, 215-225.

Higgins, S.T., Budney, A.J., Bickel, W.K. y Badger, G.J. (1994). Participation of significant others in outpatient behavioral treatment predicts greater cocaine abstinence. American Journal of General Psychiatry, 51, 568576.

Hubbard, R.L., Rachal, J.V., Craddok, S.G.Y. y Cavanaugh, E.R. (1984). Treatment outcome prospective Study (TOPS): Client characteristics and behaviors before, during and after treatment. En J.F. Tims y J.P. Ludorf (Eds), Drug abuse treatment evaluation: strategies progress and prospects. Maryland: NIDA Research Monograph, $n^{\circ} 51$. Washington, DC: US Deparment of Health and Human Services.

Joe, G.W., Simpson, D.D. y Broome, K.M: (1998). Effects of readiness for drug abuse treatment on client retention and assessment of process. Adicction, 93, 1177-90.

Kooyman, M. (1993/1996). La Comunidad Terapéutica para Drogodependientes. Bilbao. Mensajero.

Machado, M.J. y Girón, S. (1993). Perfil psicosocial y retención de heroinómanos en tratamiento ambulatorio. Adicciones, 2, 109-125.

Magura, S., Nwakeze, P.C. y Demsky, S.Y. (1998). Pre-and intreatment predictors of retention in methadone treatment using survival analysis. Addiction, 93, 51-60.

Maremmani, I., Nardini, R., Zolesi, O. y Castrogiovanni, P. (1994). Methadone dosages and therapeutic compliance during a methadone maintenance program. Drug and Alcohol Dependence, 34, 163-166.

Melnick, G., De Leon, G., Hawke, J., Jainchill, N. y Kressel, D. (1997). Motivation and readiness for therapeutic community treatment among adolescent and adult substance abusers. American Journal of Drug and Alcohol Abuse, 23, 485-506.

McCaul, M.E. y Svikis, D.S. (1991). Improving client compliance in outpatient treatment: counselor-targeted interventions. En R.W. Pickens, C.G. Leukefeld y C.R. Schuster (Eds.), Improving drug abuse treatment. NIDA Research Monograph Series, $n^{\circ}$ 106. Rockville, MD: US Department of Health and Human Services.
Morral, A.R., Belding, M.A. e Iguchi, M.Y. (1999). Identifying methadone maintenance clients at risk for poor treatment response: pretreatment and early progress indicators. Drug and Alcohol Dependence, 55, 25-33.

Ochoa, E., Arias, F., Somoza, J.C., Vicente, N. y Jordá, L. (1992). Retención en un programa de tratamiento con naltrexona: dos años de seguimiento. Adicciones, 4, 207-212.

Ochoa, E., Cebollada, A. e Ibáñez, A. (1994). Implicación familiar como predictor de un tratamiento con antagonistas opiáceos. Adicciones, 6, 51-60.

Panyard, C., Wolf, K.Y. y Dreachslin, J. (1979). Source of referral as an indicator of motivational factors and treatment outcome with drugdependent clients. International Journal of the Addictions, 5, 645-656.

Roberts, A.C. y Nishimoto, R.H. (1996). Predicting treatment retention of women dependent on cocaine. American Journal of Drug and Alcohol Abuse, 22, 313-333.

Sánchez-Carbonell, J., Camí, J. y Brigos, B. (1988). Followup of heroin addicts in Spain (EMETYST project): Results 1 year after treatment admision. British Journal of Addiction, 83, 1439-1449.

Secades Villa, R., Fernández Rodríguez, R. y Fernández Hermida, J.R. (1998). Factores asociados a la retención de heroinómanos en un programa libre de drogas. Adicciones, 10, 53-58.

Simpson, D. (1979). The relation of time spent in drug abuse treatment to posttreatment outcome. American Journal of Psychiatry, 11, 1449-1453.

Simpson, D.D. (1984). National treatment system evaluation based on the Drug Abuse Reporting Program (DARP) follow-up research. En F.M. Tims y J.P. Ludford (Eds.), Drug abuse treatment evaluation: Strategies, progress, and prospects. NIDA Research Monograph, $n^{\circ} 51$. Washington, DC: US Deparment of Health and Human Services.

Sisson, R.W. y Azrin, N.H. (1986). Family-member involvement to initiate and promote treatment of problem drinkers. Journal of Behabior Therapy and Experimental Research, 17, 15-21.

Stanton, M.D., Steier, F., Cook, L. y Tood, T.D. (1984). Narcotic detoxification in a family and home contest: Final report-1980-1983. Rockville, MD: National Institute on Drug Abuse.

Stark, M.J. (1992). Dropping out of substance abuse treatment: A clinically oriented review. Clinical Psychology Review, 12, 93-116.

Torrens, M., Castillo, C. y Perez-Sola, V. (1996). Retention in a low-threshold methadone maintenace program. Drug and Alcohol Dependence, 41, 55-59.

Tucker, J.A., Vuchinich, R.E. y Harris, C.V. (1985). Determinants of substance abuse relapse. En M. Galizio y S.A. Maisto (Eds.), Determinants of substance abuse: Biological, psychological and environmental factors. New York: Plenum Press. 
